\title{
Microfluidic applications of functionalized magnetic particles for environmental analysis: focus on waterborne pathogen detection
}

\author{
Qasem Ramadan • Martin A. M. Gijs
}

Received: 1 August 2012/Accepted: 3 August 2012/Published online: 17 August 2012

(C) Springer-Verlag 2012

\begin{abstract}
The continuous surveillance of drinking water is extremely important to provide early warning of contamination and to ensure continuous supplies of healthy drinking water. Isolation and detection of a particular type of pathogen present at low concentration in a large volume of water, concentrating the analyte in a small detection volume, and removing detection inhibiting factors from the concentrated sample, present the three most important challenges for water quality monitoring laboratories. Combining advanced biological detection methods (e.g., nucleic acid-based or immunology-based protocols) with microfluidics and immunomagnetic separation techniques that exploit functionalized magnetic particles has tremendous potential for realization of an integrated system for pathogen detection, in particular, of waterborne pathogens. Taking advantage of the unique properties of magnetic particles, faster, more sensitive, and more economical diagnostic assays can be developed that can assist in the battle against microbial pathogenesis. In this review, we highlight current technologies and methods used for realization of magnetic particle-based microfluidic integrated waterborne pathogen isolation and detection systems, which have the potential to comply in future with regulatory water quality monitoring requirements.
\end{abstract}

Keywords Hysterosalpingography - Intracervical block · Visual analog scale $\cdot$ Verbal descriptive score

Q. Ramadan $(\bowtie) \cdot$ M. A. M. Gijs

Laboratory of Microsystems, Ecole Polytechnique Fédérale

de Lausanne (EPFL), Lausanne, Switzerland

e-mail: qasem.alramadan@epfl.ch

\section{Introduction}

Contamination of drinking water with pathogens poses a significant threat to millions of people in the developing world. Even in the developed world, periodic outbreaks of diarrheal diseases are caused by protozoan parasites (Karanis et al. 2007; Bouzid et al. 2008). Therefore, a proper assessment of microbial water quality is important to provide early warning of contamination and to ensure continuous supplies of healthy drinking water. Waterborne pathogens include viruses, bacteria, and protozoa with the latter being more common than the others. The three major waterborne protozoan diseases are cryptosporidiosis, giardiasis, and amebiasis. Giardia is the most widespread protozoan causing diarrhea to 200 million symptomatic individuals worldwide (WHO 2006). The list of potential waterborne pathogens is extensive. Table 1 provides examples of waterborne pathogens.

The development of filtration and chlorination processes for treatment of drinking water virtually eliminated waterborne enteric diseases, such as typhoid and cholera in the developed world. However, microbial pathogens continue to pose a key public health challenge in providing safe drinking water. In addition to the traditional waterborne pathogens, a significant number of emerging pathogens have recently been recognized. According to the World Health Organization (WHO)'s Guidelines for Drinking-water Quality, zoonotic pathogens, which make up $75 \%$ of the emerging pathogens, pose the greatest challenges to ensuring the safety of drinking water (WHO 2011). The European Union Council Directive 98/83/EC on the quality of water requires water for human consumption to be free from any microorganisms and parasites and from any substances which pose a potential danger to human health. 
Table 1 Examples of major waterborne pathogens

\begin{tabular}{lll}
\hline Pathogen & Major disease & Ref. \\
\hline Protozoa & & \\
Cryptosporidium parvum & Cryptosporidiosis (acute diarrhea) & Szewzyk et al. (2000), Alonso et al. (2001) \\
Giardia lamblia & Giardiasis (chronic diarrhea) & Szewzyk et al. (2000) \\
Naegleria & Meningoen cephalitis & Behets et al. (2007) \\
Entamoeba histolytica & Amebic dysentery & Gerba (1996) \\
Bacteria & & \\
Salmonella & Typhoid and diarrhea & Gerba (1996) \\
Escherichia coli O157:H7 & Diarrhea can lead to hemolytic uremia syndrome & Gerba (1996) \\
Yersinia enterocolitica & Diarrhea & Gerba (1996) \\
Viruses & & \\
Hepatitis A and E & Infectious hepatitis & Straub and Chandler (2003) \\
Enteroviruses & Meningitis, paralysis, rash, fever, and diarrhea & Straub and Chandler (2003) \\
\hline
\end{tabular}

The concentration of pathogens in contaminated water varies significantly, as illustrated in Table 2 (USEPA 1988). These pathogens are normally present at very low concentration in water, which implies that there is a need of a robust and sensitive system capable of capturing and detecting these rare pathogens down to the single organism/cell level.

Typical microbial detection methods to evaluate drinking water sources start with filtration and concentration steps, aiming at isolating small concentrations of pathogens, i.e., one to 10 infectious units, from large volumes of water (40-100 L), followed by quantification of the target pathogen using a proper detection method (WHO 2011). However, concentration of the residues contained in hundreds of liters of water into less than 50 milliliters often results in the accumulation of compounds that inhibit the subsequent detection methodologies, requiring additional purification procedures to isolate target pathogens from the background interfering compounds. The problem is that additional efforts to cleanup the sample may lead to a loss of the target pathogen. Following filtration, there are a variety of options for the detection of specific pathogens including culture methodologies, microscopic observation, immunochemical approaches, and molecular methods.

Table 2 Estimated levels of enteric organisms in sewage and polluted surface water in the United States

\begin{tabular}{lll}
\hline Organism & \multicolumn{2}{l}{ Number per $100 \mathrm{ml}$} \\
\cline { 2 - 3 } & Sewage & Polluted stream water \\
\hline Coliforms & $10^{9}$ & $10^{5}$ \\
Enteric viruses & $10^{2}$ & $1-10$ \\
Giardia & 10 & $0.1-1$ \\
Cryptosporidium & $10-10^{3}$ & $0.1-10^{2}$ \\
\hline
\end{tabular}

Source USEPA (1988)
Each technique has its own particular advantages and disadvantages relative to the specific pathogen of interest. Culture methods, for example, may be an appropriate method for detection of some bacteria, but are very timeconsuming and costly for virus analysis. Likewise, protozoan pathogens and many bacterial pathogens are slowly growing and require specific growth media, making culture techniques an unlikely choice for a universal detection method. Flow cytometry allows for large volumes to be screened, but it has a detection limit of around 100 parasites per liter (Bouzid et al. 2008), so often prior filtration is required (Ferrari et al. 2006).

The danger for public health of waterborne pathogens, present even at very low concentration in drinking water, justifies the urgent need for a quantitative microbial risk assessment of infection associated with the microbial contamination of water. Success has been achieved predominantly in the downstream detection stages of the analytical process. For example, several biosensor approaches have been evaluated for the detection of waterborne protozoa, such as optical (Kramer et al. 2007), electrochemical (Setterington and Alocilja 2010), and piezoelectric (Campbell and Mutharasan 2008) methods. On the other hand, less effort was invested in sample preparation, even though it is the most time-consuming part of an analytical protocol and the major source of inaccuracy, potentially resulting in false positives/negatives or sample loss. Therefore, proper sampling and concentration techniques as well as integration of analytical process steps for pathogen detection in drinking water (sample-toanswer) should be exploited further. Despite the advancements in pathogen identification, current diagnostic methods have limitations, including laborious sample preparation, bulky instrumentation, and slow data readout. In addition, field-deployable or "point-of-interest" systems are urgently needed to facilitate detection of pathogens 
even in remote areas, before spreading of these pathogens through the public water systems occurs.

This review shows that water quality monitoring has undergone tremendous progress in recent years, with the introduction of new efficient immunological and molecular tools that offer rapid, high-throughput, sensitive, specific, and real-time detection of a wide spectrum of pathogens, competing with the traditional culture-based techniques. Important factors influencing the future potential of automated detection systems for waterborne pathogens are the increase of detection sensitivity to levels better than the detection limits required to meet public health criteria, combined with a short analysis time to provide timely alert of the risk. In order to achieve these criteria, methods for sample purification, signal amplification, and reduction of the background noise are investigated. Integration of sample preparation techniques with either immunological or molecular tools in the Lab-on-a-chip (LOC) format has a great potential to provide sensitive, specific, and quantitative analytical data on many pathogens. We discuss some of the leading enabling technologies that are of interest for integration of the analytical process and for establishing a universal and automated detection system. We will focus on the role that functionalized magnetic particles can play in nucleic acid- and immunology-based methods and highlight their potential to be employed in an integrated pathogen detection system.

\section{System requirements}

Water quality monitoring laboratories use analytical techniques with a high level of sensitivity to identify pathogenic agents. However, most of these techniques cannot be utilized in the field (e.g., in water distribution systems) or in places with poor resources, because they often require sophisticated, expensive instrumentation that needs to be used by trained personnel. Besides this, the high cost and short shelve lifetime of some reagents limit the application of the most conventional pathogen detection techniques in the field. Furthermore, despite their sensitivity, current techniques, like an enzyme-linked immunosorbent assay (ELISA), require extensive sample preparation and long readout times, which delay a prompt response and the alert in case of eventual danger. In order to realize an integrated system for waterborne pathogen detection, the following technical requirements should be satisfied:

\subsection{Integration of sample preparation and detection} processes in a semi-closed loop protocol

Depending on the dosage of the infectious agent, large sample volumes are usually required to be processed and analyzed. For example, $\sim 100 \mathrm{~mL}$ of sample is needed for bacteria and up to 1,000 L for viruses (Liu and Zhu 2005). These volumes are subsequently filtered and concentrated by membrane separation down to a volume of a few milliliters (Straub and Chandler 2003). Due to the heterogeneity of the sample matrices, secondary purification and concentration steps are required to selectively separate target pathogens from particles, debris, etc. present in the sample matrices. After finishing the sample preparation process, it is highly desirable to extract a small output volume (microliters) out of the concentrated sample, as this quantity should be compatible with downstream detection processes and, at the same time, allows reducing the amount of detection reagents and consequently the cost of the detection. In order to minimize the chance of losing any pathogen of interest, the sample preparation and detection process ideally should be conducted inside a single confined channel. In other words, the system should be able to handle a large input volume, process, and concentrate the analyte and at the same time handle a small volume during downstream detection at a high level of sensitivity, and all this should be executable in a semi-closed loop protocol. Taking into account the large differences between the volume of the initial sample; the volume after filtration and concentration; the analytical process volume; and the detection volume; and the demand for integration of the entire analysis process, from filtration to pathogen quantification, one possible scenario is to use a solid capture and sensing interface. One can position such interface within a microfluidic circuit for liquid processing, or can combine it with conventional filtration and concentration units in a stand-alone system. We will show here that using such interface, the surface of functionalized magnetic particles presents an extremely interesting option.

\subsection{Dealing with the versatility of pathogen species}

Waterborne pathogens range in size from 0.01 to $100 \mu \mathrm{m}$ in diameter (Gerba 1996), which poses enormous challenges for collection of all species from the sample. For example, the current method to capture viruses uses positively charged filters with a nominal pore size of $0.2 \mu \mathrm{m}$. Both the pore size and the charge of the filter together help in capturing the viruses. However, the same filter may not be suitable for recovery of bacteria or parasites, because it may concentrate a large amount of sediment (Straub and Chandler 2003).

2.3 Capability to overcome the matrix effect in sample collection and concentration

Isolating organisms from large volume samples is always associated with co-concentration of many types of 
particulate inhibitors that can interfere with downstream molecular and/or fluorescent detection (Schwab et al. 1996). These recovered sediments make it difficult to detect all concentrated target molecules. The system, therefore, should be able to efficiently isolate, prior to detection, the target pathogen from this complex matrix without losses to enhance the detectability of the pathogen. Depending on the source, water can contain large amounts of organic/non-organic materials. Membrane-based filtration techniques are most often used in water sample filtration. Membrane filtration, which utilizes large diameter (142 or $293 \mathrm{~mm}$ ) flat filters are used for the concentration of Cryptosporidium parvum oocysts and Giardia from water samples (Ongerth and Stibbs 1987). Water is pumped through the membrane and the retained materials are recovered by scraping the membrane, followed by centrifugation. The sample volume after centrifugation is in the range of a few milliliters (e.g., $5 \mathrm{~mL}$ ). In general, normal membrane filtration can filter $10-40$ L of low-turbidity water, while filtration of high-turbidity waters is limited to only 1-2 L. Recovery efficiencies of different parasites, however, can be influenced by the choice of the filter material. Shepherd and Wyn-Jones 1996 reported that when $100 \mathrm{~L}$ samples were seeded with 75 oocysts or 85 cysts/L, cellulose-acetate membranes gave higher recovery rates (30-40 \% for Cryptosporidium and 50-67\%, for Giardia) than polycarbonate membranes (22-36 and 41-49\%, respectively). Filtration processes are very well established and therefore will not be discussed here in detail. More details about the filtration techniques can be found in (Zarlenga and Trout 2004).

\subsection{Portability}

Existing environmental analysis systems are still not configurable as miniaturized "point-of-interest" devices. Indeed, the size of peripheral equipments required for sample preparation, such as filtration and centrifugation units, and of processing and signal acquisition equipments, such as electronic and optical components, over-shadows the small footprint of any portable system. Thus, continued miniaturization of not only the processing and detection systems, but also the peripheral equipment is important to the realization of true point-of-interest systems.

\section{Conventional water analysis methods}

\subsection{Bacteria culture and colony counting}

The culture method is the oldest bacterial detection technique and remains the standard detection method. It is highly specific but is time-consuming; since microscopy detection methods require samples containing a high amount of pathogens, they require culturing of the pathogen of interest for a few days to get sufficient pathogen enrichment. Some of the bacteria such as M. tuberculosis take 7-14 days to grow (Cheng et al. 2005). In the case of Campylobacter spec., 4-9 days are needed to obtain a negative result and between 14 and 16 days for confirmation of a positive result (Brooks et al. 2004). Some microorganisms cannot grow easily in culture which makes their identification more challenging.

\subsection{Polymerase chain reaction (PCR)}

The detection of PCR amplification products has undergone dramatic changes, since its introduction by Kary Mullis in the 1980s, which has led to revolutionary testing platforms (Mullis et al. 1986; Aw and Rose 2011). The PCR technique is based on the isolation, amplification, and quantification of a short DNA sequence of the targeted bacteria's genetic material. Examples of different PCR methods developed for bacterial detection are: (1) real-time PCR (2005), (2) multiplex PCR (Jofré et al. 2005), and (3) reverse transcriptase PCR (RT-PCR) (Deisingh and Thompson 2004). The development of real-time PCR has offered numerous advantages over conventional PCR techniques, such as a higher sensitivity and specificity, a faster rate of detection, no need for post-PCR analysis, and the capability to provide quantitative results. This technique is based on the real-time fluorescent emission by a specific dye attached to the targeted amplicon. The fluorescence intensity is proportional to the amount of amplified product (Cady et al., 2005). Based on the PCR principle, several fluorescent probes have been developed such as those found in TaqMan and molecular beaconbased protocols (Yang and Rothman 2004). Conventional PCR cannot discriminate between viable and non-viable cells because DNA is always present in dead and live cells. Reverse transcriptase PCR (RT-PCR) was developed to detect viable cells only (Yaron and Matthews 2002). The reverse transcriptase enzyme is able to synthesize singlestranded DNA from RNA. Several genes specifically present during the bacteria's growth phase can then be detected (Lazcka et al. 2007). Recently, microfluidicsbased high-density PCR systems were developed. For example, the Biotrove OpenArray system (Applied Biosystems) is capable of performing 3,072 reactions per array (Van Doorn et al. 2009; Stedtfeld et al. 2008).

\subsection{Immunology-based methods}

Immunology-based methods provide very powerful analytical tools for a wide range of targets. Immunoassays have been developed to measure the presence of an 
analyte through a specific antigen-antibody interaction. The sensitivity and specificity of an immunoassay are highly dependent on the choice of the antibodies. Among the current immune-based methods, immunomagnetic separation (IMS) emerged as one of the most powerful and reliable method for detection of pathogens, which provide an alternative method to ELISA possibly providing more rapid, sensitive, and reproducible results. IMS uses superparamagnetic particles, which are coated with antibodies against the target organisms to selectively isolate these particular organisms from the sample matrix. The IMS process starts by introducing the functionalized magnetic particles into the target-containing suspension and incubating the mixture for a period of time, which can be within the range of 30-60 min. During the incubation time, the target antigen that characterizes the organism is captured by the antibody-coated magnetic particles. The immunomagnetic complex, formed by the cells and the magnetic particles, is then separated from the suspension using a magnet. Following the magnetic separation, the isolated magnetic complex is washed repeatedly to remove unwanted contaminants and the target organisms attached with the magnetic particles, and are then transferred for further experiments or for final detection. Recently, IMS coupled with PCR showed promising results for the detection of E. coli O157:H7 (Fu et al. 2005).

\section{Microfluidics-based analysis techniques}

PCR- and immunology-based methods provide the most rapid and sensitive detection; moreover, these techniques are amenable for integration and automation and, therefore, are very promising for developing an integrated system for waterborne pathogen detection. Table 3 lists a comparison between these two methods. For more details, Velusamy et al. (2010) provide an excellent relevant comparison.

An increasing research effort has been focused on using microfluidic devices for environmental analysis, particularly pathogen detection in water and food. Several reviews have been recently published dealing with microfluidics for environmental samples. For example, Chen et al. (2006) reviewed the broad environmental applications of microchip electrophoresis coupled with electrochemical detection. Recently, Jokerst et al. (2012) published a comprehensive and focused review of the recent advances in microfluidics for environmental applications. Therefore, we will not discuss these general methods in this review, but we rather focus on magnetic particle-based assays. For more details about the wider application of microfluidics for environmental analysis applications, we refer to the above two reviews.

\subsection{Immunomagnetic-based sample preparation} methods (concentration and purification)

IMS-based methods have become the gold standard for separation and concentration of cells and biomolecules, and even for rare cell capturing. The thus obtained enriched sample can be analyzed subsequently using almost any detection method, e.g., optical, magnetic force microscopybased, plasmonic, and magnetoresistive methods among others. The fundamentals of manipulation and applications of functionalized particles and IMS were comprehensively reviewed (Gijs 2004; Gijs et al. 2010). Functionalized magnetic particles are available from a number of companies with a wide range of sizes (from a few nanometers up to a few tens of microns) that may be chosen depending on the application. IMS was already utilized to isolate pathogenic microorganisms from large sample volumes using flow-through systems. The device reported by

Table 3 Comparison between immunology-based methods and nucleic acid-based methods in pathogen detection

\begin{tabular}{|c|c|c|}
\hline & Immunology-based methods & Nucleic acid-based methods \\
\hline $\begin{array}{l}\text { Examples of } \\
\text { techniques }\end{array}$ & $\begin{array}{l}\text { ELISA (Palumbo et al. 2003); Immunomagnetic separation } \\
\text { (Hudson et al. 2001); immunochromatography strip test } \\
\text { (Shim et al. 2007) }\end{array}$ & $\begin{array}{l}\text { Real-time PCR (Rodríguez-Lázaro et al. 2005); Multiplex PCR } \\
\text { (Jofré et al. 2005); Reverse transcriptase PCR (RT-PCR) } \\
\text { (Deisingh and Thompson 2004, 2005) }\end{array}$ \\
\hline Specificity & Good (depends on available antibodies) & Excellent \\
\hline Time & 1-2 h (Mandal et al. 2011) & 6-24 h (Mandal et al. 2011; Lazcka, et al. 2007) \\
\hline $\begin{array}{l}\text { Detection } \\
\text { limit }\end{array}$ & $\begin{array}{l}\text { Single oocyst (Rheonix Inc) } 90 \% \text { recovery (Ramadan et al. } \\
\text { 2010a) }\end{array}$ & $0.2 \mathrm{cfu} / \mu \mathrm{L}$ (Beyor et al. 2009) \\
\hline Multiplexing & No data & Excellent \\
\hline $\begin{array}{l}\text { Live/dead } \\
\text { (infectivity) }\end{array}$ & No & Yes, with RT-PCR (Yaron and Matthews 2002) \\
\hline $\begin{array}{l}\text { Amenability } \\
\text { to } \\
\text { automation }\end{array}$ & Good & Good \\
\hline
\end{tabular}


Rotariu et al. (2005a, b), employed a ferromagnetic wire aligned along a fluidic silicone tube and magnetized in a uniform magnetic field. The system was utilized to recover Escherchia coli $\mathrm{O} 157$ from different carrier fluids and food homogenates with a sample volume of $50 \mathrm{~mL}$. Chandler et al. (2000) described an automated IMS system with sample preparation for isolation of Escherichia coli O157:H7 from poultry carcass rinse coupled with a nucleic acid microarray for detection. A porous $\mathrm{Ni}$ foam was used to enhance the magnetic field gradient within the flow path, providing a mechanism for immobilizing magnetic particles in the center of the flow path rather than on the tubing wall. A recovery efficiency of $32 \%$ of non-pathogenic E. coli was reported. Recovered magnetic particles were used subsequently for PCR amplification and microarray detection, and a detection limit of $10^{3} \mathrm{cfu} / \mathrm{mL}$ was reported.

Most of the current IMS devices capture the magnetic particles in a static mode, i.e., the magnetic particles are trapped against the wall of the test tube or within a porous support placed in the fluidic channel. Such static trapping works well for many applications, especially when the target analyte is abundant within the sample and when the sample contains few impurities. However, in environmental applications, the number of the target pathogens can be very small and they are hidden within a large population of impurities; therefore, a large population of magnetic particles may have to be used. As a consequence, when the sample is exposed to high magnetic field gradients during the separation process, the magnetic particles aggregate in a dense plug due to the significant dipole-dipole interactions (Sinha et al. 2009). This static trapping process results in an embedding of the analyte inside a large inhomogeneous aggregate which contains, besides the analyte, a large number of unbound magnetic particles and a large amount of impurities (e.g., sand) which will be present in different concentrations, depending on the original raw sample composition. In order to minimize the existence of undesired unbound magnetic particles and impurities, the sample should go through multiple washing steps.

Dynamic trapping of a magnetically labeled analyte using a continuous liquid flow regime in a single channel has shown to be of great potential to purify analytes from water samples for downstream detection (Ramadan et al., 2010a and b; Ramadan and Gijs 2011). Dynamic trapping of magnetic particles has also been demonstrated using an oscillatory magnetic field applied transversely to a microfluidic channel (Rida and Gijs 2004a and Moser et al. 2009b). The oscillating magnetic field enhances the interaction between the particles and the carrier fluid. The same approach has been used for on-chip mixing (Rida and Gijs 2004b) and for performing immuno-agglutination assays (Moser et al. 2009a). The superparamagnetic nature of magnetic particles allows fast magnetization and demagnetization of these particles, when they are exposed to an alternating magnetic field, such that the magnetic particles in the suspension can aggregate and disperse as a response to the applied value of the magnetic field. This special feature that superparamagnetic particles have was exploited in applications beyond magnetic separation. For example, the concept of "trapping-and-releasing" of magnetic particles was combined with continuous flow of sample and washing buffer to perform continuous washing of the sample in a fluidic channel before the analyte gets finally separated (Ramadan et al. 2010a, b; Ramadan and Gijs 2011). This mechanism was implemented to simultaneously wash and concentrate protozoan cells from different water sample matrices. The trapping-and-releasing device was realized by employing a rotating magnetic assembly beneath a microfluidic channel, which comprises an array of Neodymium Iron Boron (NdFeB) magnets that are arranged in alternate polar orientations. The rotational motion of the magnetic assembly coupled with the continuous flow of a washing buffer within the channel generates periodical trapping-and-releasing cycles, each cycle corresponding to one washing cycle (Fig. 1). The performance of the system was evaluated by isolating magnetically labeled protozoan cells from filtered, concentrated tap water, secondary effluent water, and purified water following the experimental protocols described in the US Environmental Protection Agency method (USEPA 1623 http://www.epa.gov/microbes/1623de05.pdf). A recovery efficiency of Giardia cyst of 90.5 and $18.5 \%$, from spiked tap water and secondary effluent water, respectively, and a recovery efficiency of Cryptosporidium cells of 90 and $36 \%$ from spiked tap water and secondary effluent matrix, respectively, were achieved.

Beyor et al. 2008 developed an immunomagnetic beadbased approach for isolation and preconcentration of $E$. coli from a dilute sample. The device was constructed from PDMS and glass, and incorporated on-chip pneumatic pumps for fluid flow generation. Superparamagnetic particles were loaded into the chip and held in the microchannels using an external permanent magnet. After the concentration step, off-chip PCR and capillary electrophoresis (CE) were performed. A recovery efficiency of $70 \%$ was achieved with a limit of detection 2 of cfu/mL. Similarly, Tennico and Remcho, 2010 utilized functionalized magnetic nanoparticles as the solid support for extraction of parabens in water and reported $90 \%$ analyte recovery.

Commercially available IMS kits are currently available for isolation and detection of a number of waterborne pathogens. These kits use a monoclonal antibody (mAb) against the pathogen that is covalently attached to the magnetic beads. For example, the Dynabeads ${ }^{\circledR}$ anti-Cryptosporidium IMS kit, which is approved by the USEPA, 
Fig. 1 Magnetic particle washing using the "trappingand-releasing" concept. The continuous flow of washing buffer combined with alternating trapping and releasing of the magnetic particles attached to the analyte allows periodical washing of the sample. Reprinted with permission from Ramadan et al. (2010b). Copyright 2010 Elsevier
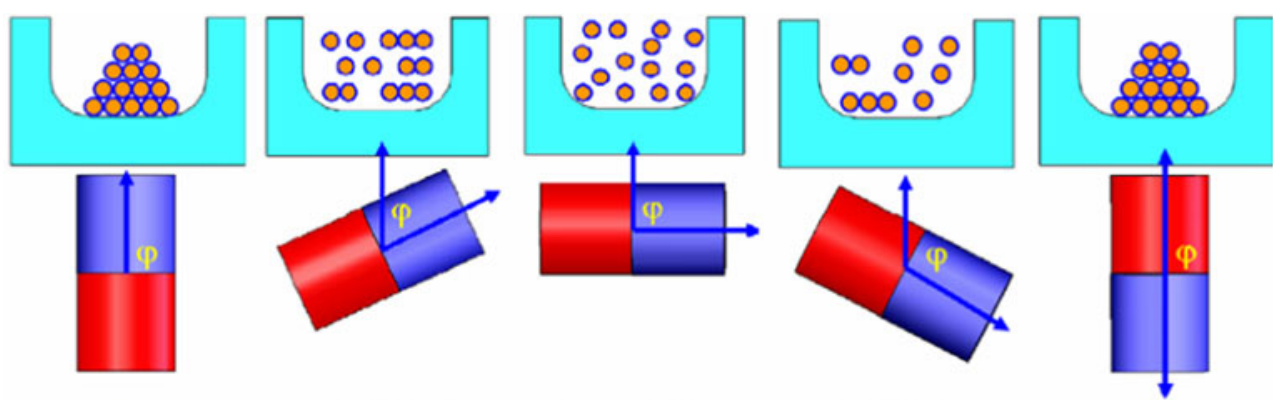

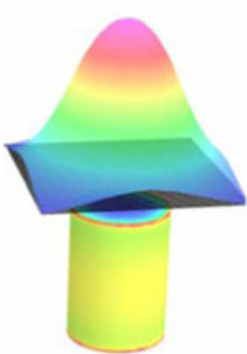

(a)

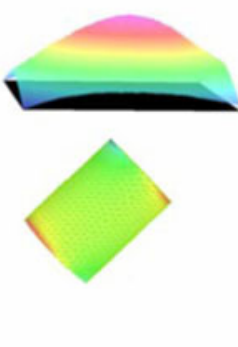

(b)

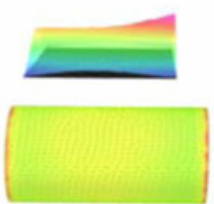

(c)

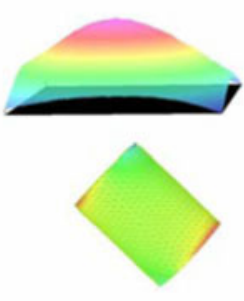

(d)

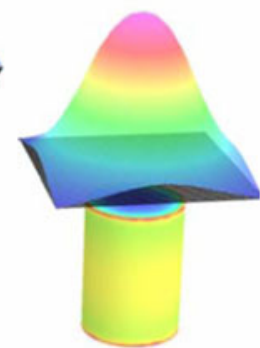

(e)

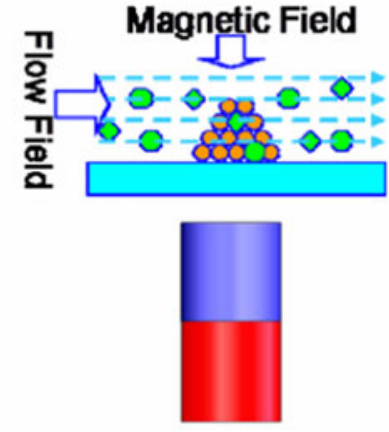

(f)

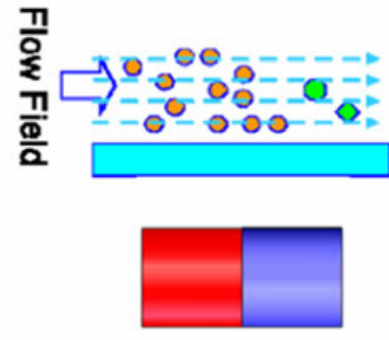

(g)

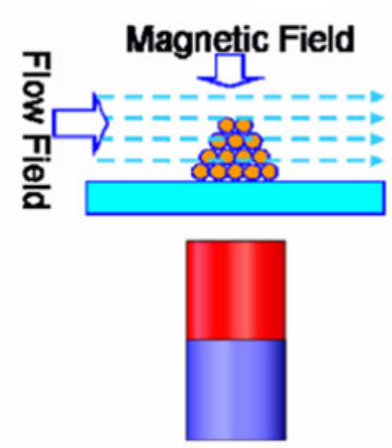

(h) consists of beads conjugated with an Immunoglobulin-M (IgM) monoclonal antibody (mAb). Another commercially available IMS kit, the Cryptoscan IMS kit (Immucell), utilizes magnetic beads conjugated with Immunoglobulin$\mathrm{G}(\mathrm{IgG}) \mathrm{mAb}$. In the IMS process, conjugated magnetic beads are mixed with the sample of interest for a certain period of time to capture the target organism. The bead conjugate-organism complex is then separated from the rest of the sample using a magnetic field. The magnetically separated bead conjugate-organism complex is then acidified using $0.1 \mathrm{M} \mathrm{HCL}$ for dissociation of the bead conjugate and the captured target pathogens.

Rheonix Inc. has developed an automated molecular diagnostic system for detecting Cryptosporidium parvum in drinking water and distinguish viable from nonviable oocysts (http://www.epa.gov/ncer/sbir/success/pdf/ rheonix.pdf; Rubina et al. 2010). Single oocyst detection was reported, but no sample volume was reported, using their developed Chemistry and Reagent Device $\left(\mathrm{CARD}^{\mathrm{TM}}\right)$. The assay was originally developed by Innovative Biotechnologies International, Inc. (IBI), prior to its acquisition by Rheonix in 2008. The process includes the following steps: (1) immunomagnetic separation and washing of oocysts; (2) heat shock induction of the hsp70 mRNA response to differentiate viable from nonviable oocysts; (3) cell lysis; (4) extraction and purification of mRNA; (5) nucleic acid sequence-based amplification (NASBA) gene amplification of the target gene sequences; and (6) detection of the NASBA amplicons on a lateral flow system utilizing liposomes, conjugated to molecular probes.

\subsection{Magnetic particle-based pathogen detection}

Besides their use in IMS applications, magnetic particles have shown great potential in detection methods that employ, for example, a magnetometer or a superconducting quantum interference device (SQUID), by recording alterations in the magnetic properties of the particles upon molecular interactions with a target (Chemla et al. 2000; Grossman et al. 2004) or with magnetic relaxometers and magnetic resonance imaging (MRI) devices, and by 
monitoring the changes in the spin-spin relaxation time (T2) of the solution's water protons due to association of a nanoparticle with a target (Kaittanis et al. 2007). Besides these magnetic property-based detection methods, IMS assays were combined with several other detection techniques, such as optical and nucleic acid-based detection, and showed great promise and a high level of efficiency and sensitivity.

\subsubsection{Magnetic particles coupled with optical detection: whole cell assay methods}

In general, optical methods are the most established and popular methods in pathogen analysis, due to the convenience they offer combined with a high selectivity and sensitivity (Mason et al. 2003; Boehm et al. 2007), with fluorescence detection being the dominant optical detection technique in microfluidic systems. Conjugation of affinity markers (e.g., antibodies, DNA, etc.) with fluorescent compounds like fluorescein isothiocyanate (FITC), phycoerythrin (PE), and Alexa-dyes is most commonly used. Other optical methods include chemiluminescence (CL), bioluminescence (BL), and Surface Plasmon Resonance (SPR) biosensors. Incorporating conventional optical or electrochemical devices with microfluidic detection systems has been widely explored (Rider et al. 2003; Schafer et al. 2009; Cheng et al. 2007).

In the USEPA 1623 standard method, the IMS process is typically followed by fluorescent antibody labeling of the concentrated samples. Then the recovered and stained target-containing sample is spread on a microscope slide and the labeled targets are counted under an optical microscope. Besides the IMS process, optical microscopic cell counting possesses the most operational challenges due to the long processing time, the necessity of skilled personnel, combined with the high possibility of producing errors. Aligned with the requirement of obtaining accurate results in the shortest possible time, it is desirable to implement the standard method using an automated system that minimizes human interference and reduces possibilities of inducing errors. Several efforts have been made to develop microfluidics-based cell counting devices by integrating fluorescence detection into the microfabricated chips (Wang et al. 2008; Lin et al. 2008). Rodriguez et al. (2005) developed a prototype for counting of CD4 cells by capturing the stained cells on a membrane within a microchip followed by imaging the captured cells and converting the digital image into a cell count using a computer algorithm. However, the captured cells were randomly distributed on the surface/membrane, which may result in random cell clustering. In the case of a small number of cells in large sample volume, such as waterborne pathogens, this may sharply reduce the counting accuracy. In order to avoid miss-counting, it is desirable to arrange the captured cells on individual sites in a cell array to facilitate digital imaging-based automated counting process. For example, micro-arraying of a large population of cells has been demonstrated for hydrodynamically employing microfabricated well arrays (Rettig and Folch 2005). However, the aim of this arraying method was not to count the cells and it was not necessary to capture all the cells introduced into the system.

Arraying all individual cells out of a complete captured cell population was demonstrated by conjugating magnetic particles to Giardia lamblia cysts, which were recovered from a water sample using IMS and treated with alternate trapping-and-releasing steps to finally position the target organisms in an individual array (Ramadan 2009). The magnetic arraying device was combined with planar microscale current-carrying conductors and a permanent magnet to generate periodically arranged magnetic attracting and repelling zones. The periodic trapping and releasing of the target organisms ensured single cell immobilization in each trapping zone, enhanced the capture efficiency, and, at the same time, avoided trapping of more than one cell in any of the trapping zones.

Cellular arrays facilitate cell counting either by direct counting through the microscope or by imaging the array, followed by image processing. A continuous effort, therefore, has been directed to miniaturization of imaging systems that minimize human factor-related errors and to reduce the dependence of optical detection on bulky optical systems such as a fluorescence microscope. Recently, the Ozcan group at the University of California at Los Angeles demonstrated a compact imaging system using a cell phone-based imager (Tseng et al. 2010; Zhu et al. 2011a, b). The fluorescent emission from the sample was imaged using an integrated lens that was positioned in front of the lens of a cell-phone camera. The performance of this device was validated by imaging various fluorescent microobjects including Giardia lamblia cysts over a large fieldof-view of $81 \mathrm{~mm}^{2}$ with a spatial resolution of $10 \mu \mathrm{m}$. Integrating such a compact and cost-effective fluorescent imaging system and data wireless transmission into a water quality monitoring platform could be very useful for achieving an integrated solution for water quality monitoring, which, moreover, has the potential to transmit the acquired images obtained from remote water resource environments to a central lab.

Other optical detection methods that utilize magnetic particles include plasmonics, surface-enhanced Raman spectroscopy (SERS), and Giant Magnetoresistance (GMR). For example, Zhang et al. (2012) developed a magnetic-plasmonic $\mathrm{Fe}_{3} \mathrm{O}_{4}$ - $\mathrm{Au}$ core-shell nanoparticles (Au-MNPs) platform which enabled fast concentration, detection, and differentiation of bacterial cells. The 
Au-MNPs were concentrated (condensed) to a small dot by applying an external magnetic field to a point on the surface (Fig. 2). The small dot exhibited a strong SERS effect because of the plasmonic property offered by the starshaped gold nanoshells and the high density of the spots formed by the closely packed Au-MNPs. The latter provided a highly sensitive SERS substrate for detection of molecules with a reduced limit of detection. The strong SERS spectra of the bacteria also enabled accurate differentiation of bacterial strains. Similarly, Wang and Irudayaraj (2010) demonstrated site-selective and tunable assembly of $\mathrm{Fe}_{3} \mathrm{O}_{4}$ nanoparticles onto gold nanorods of different aspect ratios with tunable plasmonic and magnetic properties. The fabricated $\mathrm{Fe}_{3} \mathrm{O}_{4}$-Au rod probes of different aspect ratios were functionalized with relevant antibodies and used in simultaneous optical detection, magnetic separation, and thermal ablation of multiple pathogens such as E. coli and $S$. typhimurium. Mujikaa et al. (2009) reported detection and quantification of Escherichia coli O157:H7 in food and clinical samples using a GMR sensor in a three-dimensional microfluidic network. Lee et al. (2008) showed sensitive and rapid detection of Staphylococcus aureus using a miniaturized diagnostic magnetic resonance system and magnetic NPs to amplify molecular interactions.

\subsubsection{Magnetic particles coupled with nucleic acid detection (PCR assays)}

Instead of searching for the entire microorganism, as was the case for previously discussed immunology-based methods, molecular detection methods look for genes and proteins, because the specificity of an organism can be distinguished at the genomic level, or by alterations in the gene expression and protein modifications (Salyers and Whitt 2002). PCR relies on repeated thermal cycling of the sample in different temperature zones using the following basic sequence: denaturation to single-stranded DNA, annealing primers to the single-stranded DNA template, and polymerase extension of the annealed duplex DNA. Nucleic acid detection has proven to be very sensitive and specific due to target amplification and base-pairing interactions. DNA-based pathogen detection can be achieved by direct target probing or post-capture target amplification. Direct target probing using hybridization-based assays is limited in terms of sensitivity, thus requiring additional signal enhancement techniques. One of the enhancement techniques includes choosing for a bead-based method, which reduces diffusion time and increases the probability of realizing a biorecognition event (Mairhofer et al. 2009). In addition, it is easier to detect small numbers of microparticles rather than fluorophore molecules or nanoparticles, as individual microparticles can be easily counted using routine optical microscopy (Mulvaney et al. 2004) or by magnetic detection (Rife et al. 2003). The combination of microfluidics and magnetoelectronics enabled fast and highly sensitive and specific multiplexed detection (Mulvaney et al. 2007), whereas miniaturization of PCR allowed rapid thermal cycling and permitted the use of small sample quantities, and has good potential of integration with other microfluidics-based processes. In addition, magnetic nanoparticle conjugates of nucleic acids have been designed as probes for the fast identification of several pathogens (Kaittanis et al. 2010).

Zhao et al. (2006) demonstrated heat shock stimulation, magnetic separation, RT-PCR, and electrochemical detection using discrete modules. The mRNA products associated with the protein complex GroEL, one of the well-known heat shock proteins in E. coli, was studied as an indicator for viability (Fig. 3). Beyor et al. (2009) integrated cell preconcentration, purification, PCR, and capillary
Fig. 2 Schematics of the condensation process of AuMNPs and bacteria (left) and the biomolecular characteristics of the bacterial cell wall that can possibly be detected by SERS (right). Reprinted with permission from Zhang et al. (2012). Copyright 2011 Elsevier

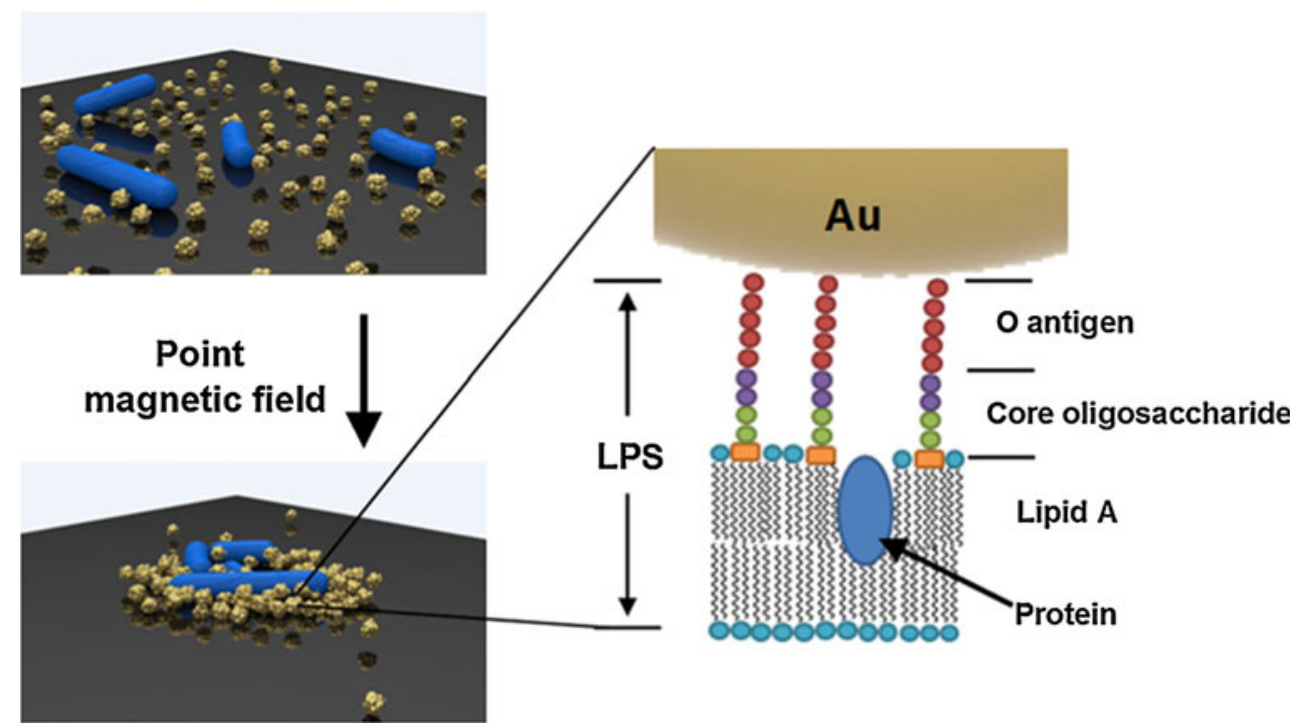




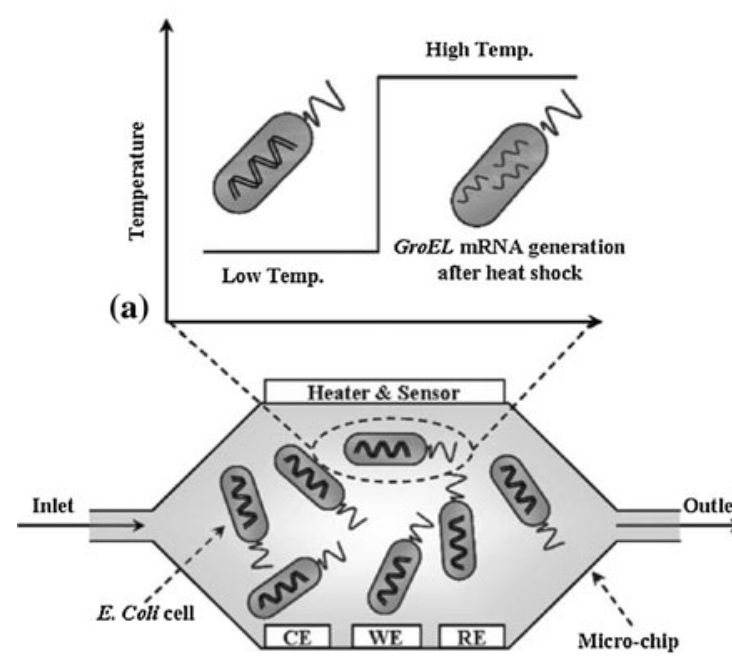

Fig. 3 mRNA associated with the expression of the protein complex GroEL is hybridized with a sequence-specific biotinylated capturing probe and then directly isolated from the cell lysates using streptavidin-modified magnetic particles. The mRNA is amplified by RT-PCR with biotin-labeled primer and the cDNA amplicon is

electrophoresis (CE) analysis in a single microfluidic chip. Functionalized magnetic particles were immobilized by an external magnet in the capture channels and the sample solution was driven through the magnetic particle bed. After capture and washing, the particle-cell duplexes were pumped into the PCR reactor using on-chip pumps for DNA amplification. The resulting PCR products of Escherichia coli K12 and $E$. coli $O 157$ targets were then electrophoretically separated and detected. A detection limit of $0.2 \mathrm{cfu} / \mu \mathrm{L}$ was reported with an input volume of $50 \mu \mathrm{L}$. LeBlanc et al. (2009) developed a method for multiplexed detection of viral pathogens, using pestiviruses as a model. Pestivirus RNAs were isolated and amplified using RT-PCR. Subsequently, PCR products were hybridized on a DNA capture microarray, and streptavidin-coated superparamagnetic particles were applied and attracted to the slide surface via magnetic force to allow rapid and simple visualization.

Furthermore, magnetic particles were incorporated into many other microfluidic systems as a mobile solid support for pathogen DNA/RNA manipulation, processing, and detection. For example, Lien et al. (2011) developed a magnetic bead-based fluorescent immunoassay which used monoclonal antibody $(\mathrm{mAb})$-conjugated immunomagnetic beads, and as a target influenza A viral particles (A/H1N1 and $\mathrm{A} / \mathrm{H} 3 \mathrm{~N} 2$ ). The magnetic complex was labeled by a specific mAb linked to R-phycoerythrin (PE), and the optical intensity of the magnetic complexes was analyzed by a fluorescence microscope. Zaytseva et al. (2005) used two sets of DNA probes that hybridized specifically with the viral target RNA. A generic probe (reporter probe), which was coupled to liposomes that encapsulated

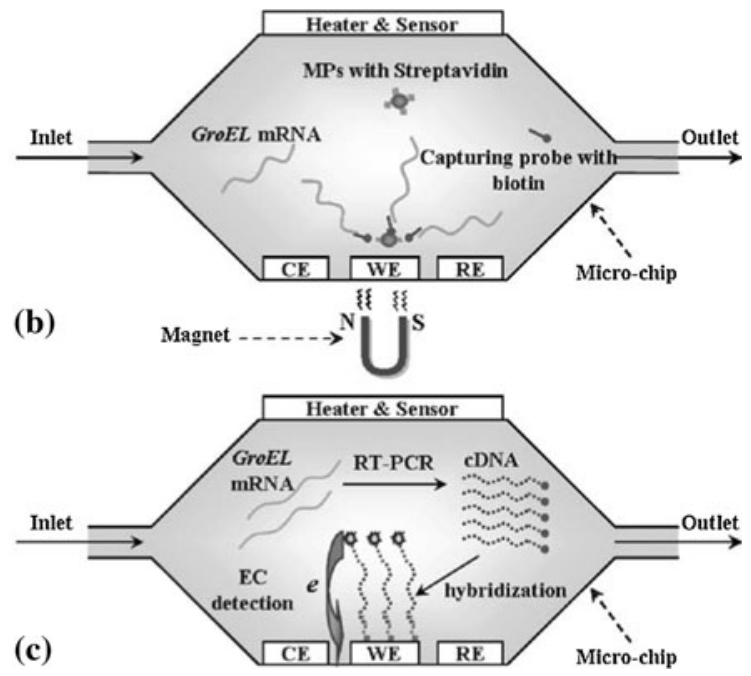

then hybridized to the immobilization probe on a working electrode surface. The biotin end of amplicon is further modified with streptavidin gold nanoparticles followed by detection of electrochemical signals of silver oxidation/reduction on gold. Reprinted with permission from Zhao et al. (2006). Copyright 2006 Elsevier

fluorescent dye molecules, was designed to bind to four Dengue virus serotypes, and four specific probes (capture probes) were designed to bind to the specific serotype only. The specific probes were immobilized on the surface of magnetic beads via biotin-streptavidin interaction. Target RNA was amplified using nucleic acid sequence-based amplification (NASBA). Liposomes with reporter probes and beads with capture probes were incubated with amplified targets prior to introduction of the mixture into a microchannel, where the sandwich complexes were subsequently captured on the magnet and detected by means of fluorescence microscopy. Chang et al. (2012) reported an integrated microfluidic system for detection of aquaculture pathogens, which contained microvalves, micropumps, reaction chambers, and washing units, such that the process for isolation of pathogen DNA, nucleic acid amplification, and optical detection was performed in automated manner. The DNA of the target pathogen was first isolated by magnetic beads coated with specific nucleotide probes after cell lysis. Then the extracted DNA fragments were amplified by a loop-mediated isothermal amplification process and the amplified products were detected optically.

\section{Toward a "sample-to-answer" system for waterborne pathogens detection}

The wide spectrum of waterborne pathogens and the extremely low concentration of the infectious pathogens in water justify the urgent need for an integrated quality monitoring system, in particular for pathogen detection. 
The water quality detection objectives coupled with the low concentrations of etiological agents in water implies that the concentration method must be able to capture these rare pathogens from very large volume samples and with minimal loss. Besides the low number of expected pathogens in the original water sample, the filtered and eluted sample normally contains a high abundance of various kinds of particulates such that the rare pathogens (if existing) become embedded in a complex matrix, which significantly influences any downstream detection process. Therefore, preparative cleanup and concentration steps are required to prevent the existing particulates from obscuring the target pathogen detection and quantification. Integration of sample preparation and detection processes in a single device could be a key factor satisfying all requirements. With this strategy, the processing of a water sample is conducted inside a single confined channel, within which all the sample preparation, processing, and detection processes are performed, avoiding manual/robotic-assisted transfer of sample between several vessels. Such system would significantly reduce the chances for target loss during the sample manipulation processes and has the potential of transfer of the sample analysis from the central lab to the point-of-interest.

Realization of such a system requires tackling many challenging issues, as discussed above, and implies integrating disparate techniques and detection methods in one platform. In other words, the challenge is the integration of biochemistry and instrumentation, which includes sample collection, sample preparation, detection and data analysis, and readout functions. An ideal system would be able to provide an automated and rapid detection, identification, enumeration, and characterization of the target organism from complex matrices in a high throughput manner and with a high sensitivity and specificity of the detection. The system should also be able to simultaneously detect viruses, bacteria, and protozoa. The routine test should be performed at low cost using, for example, a disposable chip/cartridge. Finally, the system should be automatically (unattended) operated on the site of interest. Based on the literature reviewed above, an integrated system can be conceived, as schematically shown in Fig. 4. This diagram proposes either an immunology- or nucleic acid-based detection method and highlights the possible role of functionalized magnetic particles in the different processing steps, from sample preparation to detection and enumeration. The proof of concept of such integrated fluidic cartridge for two protozoa (Giardia lamblia and Cryptosporidium spec.) isolation and detection using IMS, followed by cell arraying and fluorescence-based quantification was demonstrated (unpublished data). Even though the pathogen recovery and detection were implemented separately, an integrated microfluidic cartridge, which contained a fluidic network, valves, and a magnetic particle-based cell arraying chip was fabricated using injection molding (Fig. 5).

\section{Concluding remarks}

We have shown several technologies and methods that could be incorporated into a single integrated waterborne pathogen isolation and detection system, which has the potential of multiplexed detection of a wide range of pathogens, possibly satisfying the regulatory water quality monitoring requirements. However, while significant research efforts have been made on developing efficient detection tools from artificial pure or spiked samples, relatively little attention has been focused on sample

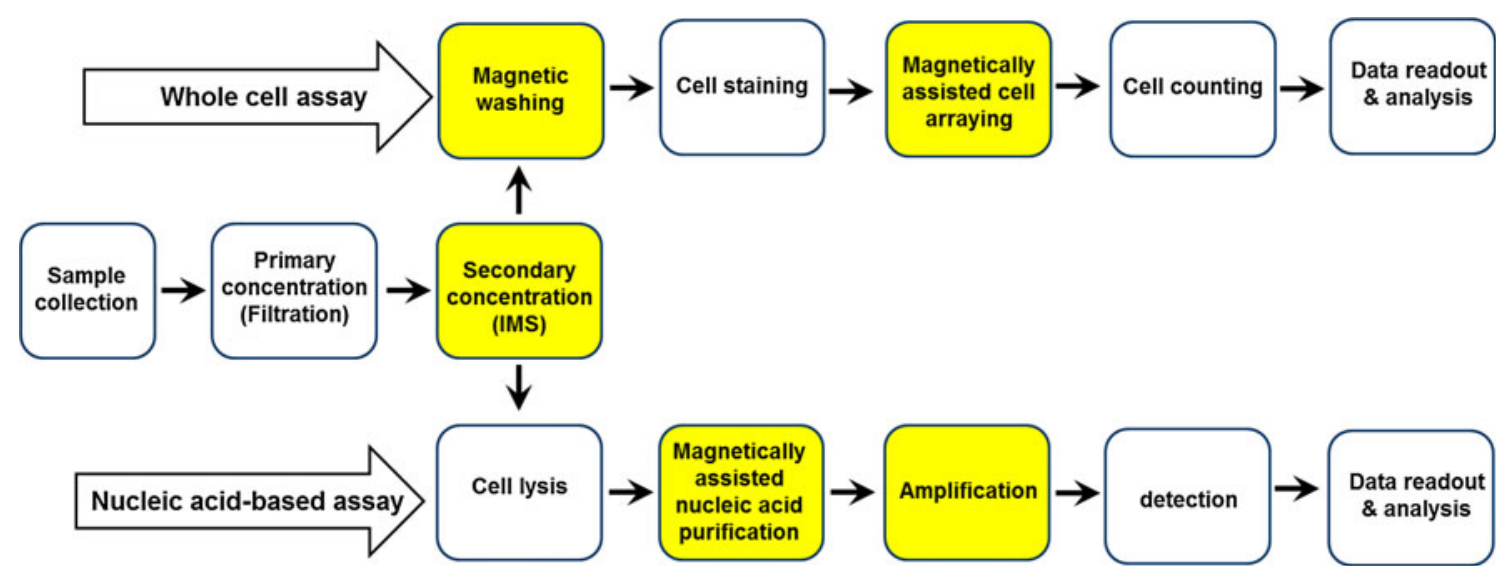

Fig. 4 Block diagram of an integrated system for waterborne detection with all its processes and functionalities, from sample preparation to detection. Two potential approaches are proposed: immunology-based whole cell detection and nucleic acid-based detection. Magnetic particles play a crucial role in the major steps in each method, as highlighted. Adopting functionalized magnetic particles in these approaches clearly facilitates automating the biochemical protocols and integrating the different functions of each detection method 


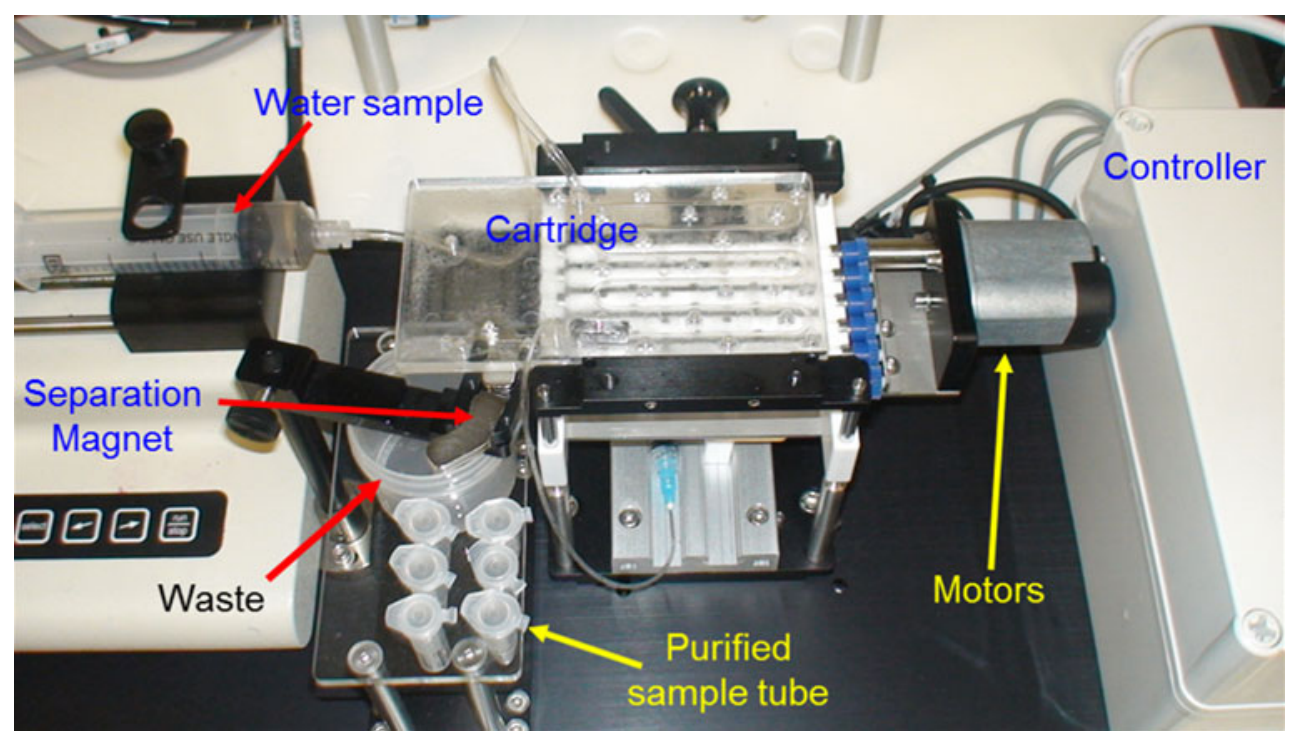

Fig. 5 Microfluidic setup with an injection-molded fluidic cartridge for waterborne pathogen isolation and detection. Reprinted with permission from Ramadan et al. (2010). Copyright 2010 Elsevier

preparation and even less attention has been given to system-level design. We have shown that functionalized magnetic particles can play significant roles in both the sample preparation and detection processes. The great advances in magnetic particles synthesis and functionalization, the possibility to manipulate and array these particles with high spatial resolution, and the combination of cell-based or molecular techniques and advanced optics coupled with microfluidic technology offer great perspectives to bring an integrated system of pathogen detection into reality. Such integrated system not only would allow reducing the footprint of the analytical platform and offering portability, but also would accelerate the interactions between the target analyte and the sensing element (e.g., the functionalized magnetic particles), which would be beneficial to the sensitivity of pathogen detection.

\section{References}

Alonso JL, Amoros I, Canigral I (2001) Development and evaluation of a real-time PCR assay for quantification of Giardia and Cryptosporidium in sewage samples. Appl Microbiol Biotechnol 89:1203-1211

Aw TG, Rose JB (2011) Detection of pathogens in water: from phylochips to qPCR to pyrosequencing. Cur Opin Biotechnol 23:1-9

Behets J, Declerck P, Delaedt Y, Verelst L, Ollevier F (2007) A duplex real-time PCR assay for the quantitative detection of Naegleria Fowleri in water samples. Water Res 41:118-126

Beyor N, Seo TS, Liu P, Mathies RA (2008) Immunomagnetic beadbased cell concentration microdevice for dilute pathogen detection. Biomed Microdevices 10:909-917

Beyor N, Yi L, Seo TS, Richard A (2009) Mathies. Integrated capture, concentration, polymerase chain reaction, and capillary electrophoretic analysis of pathogens on a chip. Anal Chem 81:3523-3528

Boehm DA, Gottlieb PA, Hua SZ (2007) On-chip microfluidic biosensors for bacterial detection and identification. Sens Actuat B 126:508-514

Bouzid M, Steverding D, Tyler MK (2008) Detection and surveillance of waterborne protozoan parasites. Curr Opin Biotechnol 19:1-5

Brooks BW, Devenish J, Lutze-Wallace CL, Milnes D, Robertson RH, Berlie-Surujballi G (2004) Evaluation of a monoclonal antibody-based enzyme-linked immunosorbent assay for detection of Campylobacter fetus in bovine preputial washing and vaginal mucus samples. Vet Microbiol 103:77-84

Cady NC, Stelick S, Kunnavakkam MV, Batt CA (2005) Real-time PCR detection of Listeria monocytogenes using an integrated microfluidics platform. Sens Actuat B 107:332-341

Campbell GA, Mutharasan R (2008) Near real-time detection of Cryptosporidium parvum oocyst by IgM-functionalized piezoelectric-excited millimeter-sized cantilever biosensor. Biosens Bioelectron 23:1039-1045

Chandler DP, Brown J, Call DR, Grate JW, Holman DA, Olson L, Stottlemyer MS (2000) Continuous, automated immunomagnetic separation and microarray detection of E. coli O157:H7 from poultry carcass rinse. Int J Food Microbiol 70:143-154

Chang WH, Yang SY, Wang CH, Tsai MA, Wang PC, Chen TY, Chen SC, Lee GB (2012) Rapid isolation and detection of aquaculture pathogens in an integrated microfluidic system using loop-mediated isothermal amplification. Sens Actuat B. doi: 10.1016/j.snb.2011.12.054

Chemla YR, Grossman HL, Poon Y, McDermott R, Stevens R, Alper MD, Clarke J (2000) Ultrasensitive magnetic biosensor for homogeneous immunoassay. PNAS 97:14268-14272

Chen G, Lin Y, Wang J (2006) Monitoring environmental pollutants by microchip capillary electrophoresis with electrochemical detection. Talanta 68:497-503

Cheng VCC, Yew WW, Yuen KY (2005) Molecular diagnostic in turberculosis. Eur J Clin Microbiol Infect Dis 24:711-720

Cheng XH, Liu YS, Irimia D, Demirci U, Yang L, Zamir L, Rodríguez WR, Toner Mand Bashir R (2007) Cell detection and counting through cell lysate impedance spectroscopy in microfluidic devices. Lab Chip 7:746-755 
Deisingh AK, Thompson M (2004) Strategies for the detection of Escherichia coli O157:H7 in foods. J Appl Microbiol 96:419-429

Ferrari BC, Stoner K, Bergquist PL (2006) Applying fluorescence based technology to the recovery and isolation of Cryptosporidium and Giardia from industrial wastewater streams. Water Res 40:541-548

Fu Z, Rogelj S, Kieft TL (2005) Rapid detection of Escherichia coli O157:H7 by immunomagnetic separation and real-time PCR. Int J Food Microbiol 99:47-57

Gerba CP (1996) Pathogens in the environment. In: Brusseau ML, Pepper IL, Gerba CP (eds) Pollution science. Academic Press, New York, pp 279-299

Gijs MAM (2004) Magnetic bead handling on-chip: new opportunities for analytical applications. Microfluid Nanofluid 1:22-40

Gijs MAM, Lacharme F, Lehmann U (2010) Microfluidic applications of magnetic particles for biological analysis and catalysis. Chem Rev 110:1518-1563

Grossman HL, Myers WR, Vreeland VJ, Bruehl R, Alper MD, Bertozzi CR, Clarke J (2004) Detection of bacteria in suspension by using a superconducting quantum interference device. PNAS 101:129-134

http://www.epa.gov/microbes/1623de05.pdf

Hudson JA, Lake RJ, Savill MG, Scholes P, McCormick RE (2001) Rapid detection of Listeria monocytogenes in ham samples using immunomagnetic separation followed by polymerase chain reaction. J Appl Microbiol 90:614-621

Jofré A, Martin B, Garriga M, Hugas M, Pla M, Rodríguez-Lázaro D, Aymerich T (2005) Simultaneous detection of Listeria monocytogenes and Salmonella by multiplex PCR in cooked ham. Food Microbiol 22:109-115

Jokerst JC, Emory JM, Henry CS (2012) Advances in microfluidics for environmental analysis. Analyst 137:24-34

Kaittanis C, Naser SA, Perez JM (2007) One-step, nanoparticlemediated bacterial detection with magnetic relaxation. Nano Lett $7: 380-383$

Kaittanis C, Santra S, Perez JM (2010) Emerging nanotechnologybased strategies for the identification of microbial pathogenesis. Adv Drug Deliver Rev 62:408-423

Karanis P, Kourenti C, Smith H (2007) Waterborne transmission of protozoan parasites: a worldwide review of outbreaks and lessons learnt. J Water Health 5:1-38

Kramer MF, Vesey G, Look NL, Herbert BR, Simpson-Stroot JM, Lim DV (2007) Development of a Cryptosporidium oocyst assay using an automated fiber optic-based biosensor. J Biol Eng 1:3

Lazcka O, Del Campo FJ, Munoz FX (2007) Pathogen detection. A perspective of traditional methods and biosensors. Biosen Bioelect 22:1205-1217

LeBlanc N, Gantelius J, Schwenk JM, Stahl K, Blomberg J, Andersson-Svahn H, Belaka S (2009) Development of a magnetic bead microarray for simultaneous and simple detection of four pestiviruses. J Virol Meth 155:1-9

Lee H, Sun E, Ham D, Weissleder R (2008) Chip-NMR biosensor for detection and molecular analysis of cells. Nat Med 14:869-874

Lien KY, Hung LY, Huang TB, Tsai YC, Lei HY, Lee GB (2011) Rapid detection of influenza A virus infection utilizing an immunomagnetic bead-based microfluidic system. Biosens Bioelectron 26:3900-3907

Lin CC, Chen A, Lin CH (2008) Microfluidic cell counter/sorter utilizing multiple particle tracing technique and optically switching approach. Biomed Microdevices 10:55-63

Liu WT, Zhu L (2005) Environmental microbiology-on-a-chip and its future impacts. Trends Biotechnol 23:174-179

Mairhofer J, Roppert K, Ertl P (2009) Microfluidic systems for pathogen sensing: a review. Sensors 9:4804-4823

Mandal PK, Biswas AK, Choi K, Pal UK (2011) Methods for rapid detection of foodborne pathogens: an overview. Am J Food Technol 6:87-102
Mason HY, Lloyd C, Dice M, Sinclair R, Ellis W, Powers L (2003) Taxonomic identification of microorganisms by capture and intrinsic fluorescence detection. Biosens Bioelectron 18:521-527

Moser Y, Lehnert T, Gijs MAM (2009a) On-chip immuno-agglutination assay with analyte capture by dynamic manipulation of superparamagnetic beads. Lab Chip 9:3261-3267

Moser Y, Lehnert T, Gijs MAM (2009b) Quadrupolar magnetic actuation of superparamagnetic particles for enhanced microfluidic perfusion. Appl Phys Lett 94:022505

Mujikaa M, Arana S, Castano E, Tijero M, Vilares R, Ruano-López J, Cruzc A, Sainz L, Berganza J (2009) Magnetoresistive immunosensor for the detection of Escherichia coli O157:H7 including a microfluidic network. Biosens Bioelect 24:1253-1258

Mullis K, Faloona F, Scharf S, Saiki R, Horn G, Erlich H (1986) Specific enzymatic amplification of DNA in vitro: the polymerase chain reaction. In: Cold spring harbor symposia on quantitative biology, vol 51. Cold Spring Harbor Laboratory, Cold Spring Harbor, NY, pp 263-273

Mulvaney SP, Mattoussi HM, Whitman LJ (2004) Incorporating fluorescent dyes and quantum dots into magnetic microbeads for immunoassays. Biotechniques 36(4):602-606 (608-609)

Mulvaney SP, Cole CL, Kniller MD, Malito M, Tamanaha CR, Rife JC, Stanton MW, Whitman LJ (2007) Rapid, femtomolar bioassays in complex matrices combining microfluidics and magnetoelectronics. Biosens Bioelectron 23:191-200

Ongerth JE, Stibbs HH (1987) Identification of Cryptosporidium oocysts in river water. Appl Environ Microbiol 53:672-676

Palumbo JD, Borucki MK, Mandrell RE, Gorski L (2003) Serotyping of Listeria monocytogenes byenzyme-linked immunosorbent assay and identification of mixed-serotype cultures by colony immunoblotting. J Clin Microbiol 41:564-571

Ramadan Q (2009) Reversible assembly of magnetized particles: application to water-borne pathogen enumeration. J App Phys 106(124701):2009

Ramadan Q, Gijs MAM (2011) Simultaneous sample washing and concentration using a "trapping-and-releasing" mechanism of magnetic beads on a microfluidic chip. Analyst 136:11571166

Ramadan Q, Lau TT, Ho S (2010a) Magnetic-based purification system with simultaneous sample washing and concentration. Anal Bioanal Chem 396:707-714

Ramadan Q, Lay C, Teo W, Hua FH (2010b) Flow-through immunomagnetic separation system for waterborne pathogens isolation and detection: application to giardia and cryptosporidium cells isolation. Anal Chim Acta 673:101-108

Rettig JR, Folch A (2005) Large-scale single-cell trapping and imaging using microwell arrays. Anal Chem 77:5628-5634

Rida A, Gijs MAM (2004a) Dynamics of magnetically retained supraparticle structures in a liquid flow. Appl Phys Lett 85:4986-4988

Rida A, Gijs MAM (2004b) Manipulation of self-assembled structures of magnetic beads for microfluidic mixing and assaying. Anal Chem 76:6239-6246

Rider TH, Petrovick MS, Nargi RE, Harper JD, Schwoebel ED, Mathews RH, Blanchard DJ, Bortolin LT, Young AM, Chen J, Hollis MA (2003) A B cell-based sensor for rapid identification of pathogens. Science 301:213-215

Rife JC, Miller MM, Sheehan PE, Tamanaha CR, Tondra M, Whitman LJ (2003) Design and performance of GMR sensors for the detection of magnetic microbeads in biosensors. Sens Actuat A 107:209-218

Rodriguez WR, Christodoulides N, Floriano PN, Graham S, Mohanty S, Dixon M, Hsiang M, Peter T, Zavahir S, Thior I, Romanovicz D, Bernard B, Goodey AP, Walker BD, McDevitt JT (2005) A microchip CD4 counting method for HIV monitoring in resource-poor settings. PLoS Med 2:e182 
Rodríguez-Lázaro D, D’Agostino M, Herrewegh A, Pla M, Cook N, Ikonomopoulos J (2005) Real-time PCR-based methods for detection of Mycobacterium avium subsp. paratuberculosis in water and milk. Int J Food Microbiol 101:93-104

Rotariu O, Ogden ID, MacRae M, Badescu V, Strachan NJC (2005a) A immunomagnetic separator for concentration of pathogenic micro-organisms from large volume samples. J Magnet Magnet Mater 293:589-596

Rotariu O, Ogden ID, MacRae M, Udrea LE, Strachan NJC (2005b) Multiple sample flow through immunomagnetic separator for concentrating pathogenic bacteria. Phys Med Biol 50:2967-2977

Rubina Y, Chen Z, Whitney H, Gwendolyn S, Montagna R (2010) Automatic and rapid molecular detection of $\mathrm{E}$. coli and Enterococci in raw recreational water samples using the fully automated Rheonix CARD ${ }^{\circledR}$ Technology Platform. Great Lakes beach conference meeting, October 2010

Salyers AA, Whitt DD (2002) Bacterial pathogenesis: a molecular approach, 2nd edn. ASM Press, Washington, DC

Schafer DN, Gibson EA, Salim EA, Palmer AE, Jimenez R, Squier J (2009) Microfluidic cell counter with embedded optical fibers fabricated by femtosecond laser ablation and anodic bonding. Opt Express 17:6068-6073

Schwab KJ, DeLeon R, Sobsey MD (1996) Immunoaffinity concentration and purification of waterborne enteric viruses for detection by reverse transcriptase PCR. Appl Environ Microbiol 62:2086-2904

Setterington EB, Alocilja EC (2010) Rapid electrochemical detection of polyaniline-labeled Escherichia coli O157:H7. Biosens Bioelectron 26:2208-2214

Shepherd KM, Wyn-Jones AP (1996) An evaluation of methods for the simultaneous detection of Cryptosporidium oocysts and Giardia cysts from water. Appl Environ Microbiol 62:1317-1322

Shim WB, Choi JG, Kim JY, Yang ZY, Lee KH, Kim MG DO, Ha SD, Kim KS, Kim KY, Kim CH, Ha KS, Eremin SA, Chung AD (2007) Production of monoclonal antibody against Listeria monocytogenes and its application to immunochromatography strip test. J Microbiol Biotechnol 17:1152-1161

Sinha A, Ganguly R, Puri K (2009) Magnetic separation from superparamagnetic particle suspensions. J Magn Magn Mater 321:2251-2256

Stedtfeld RD, Baushke SW, Tourlousse DM, Miller SM, Stedtfeld TM, Gulari E, Tiedje JM, Hashsham SA (2008) Development and experimental validation of a predictive threshold cycle equation for quantification of virulence and marker genes by high-throughput nanoliter-volume PCR on the OpenArray Platform. Appl Environ Microbiol 74:3831-3838

Straub T, Chandler D (2003) Towards a unified system for detecting waterborne pathogens. J Microbiol Methods 53:185-197

Szewzyk U, Szewzyk R, Manz W, Schleifer KH (2000) Microbiological safety of drinking water. Annu Rev Microbiol 54:81-127
Tseng D, Mudanyali O, Oztoprak C, Isikman SO, Sencan I, Yagliderea O, Ozcan A (2010) Lensfree microscopy on a cellphone. Lab Chip 10:1787-1792

United States Environmental Protection Agency (1988) Comparative health effects assessment of drinking water. Washington, DC

Van Doorn R, Klerks MM, van Gent-Pelzer MPE, Speksnijder AGCL, Kowalchuk GA, Schoen CD (2009) Accurate quantification of microorganisms in PCR-inhibiting environmental DNA extracts by a novel internal amplification control approach using Biotrove OpenArrays. Appl Environ Microbiol 75:7253-7260

Velusamy V, Arshak K, Korostynska O, Oliwa K, Adley C (2010) An overview of foodborne pathogen detection: in the perspective of biosensors. Biotechnol Adv 28:232-254

Wang C, Irudayaraj J (2010) Multifunctional magnetic-optical nanoparticle probes for simultaneous detection, separation, and thermal ablation of multiple pathogens. Small 6:283-289

Wang YN, Kang Y, Xu D, Chan CH, Barnett L, Kalams SA, Lib D, Li DQ (2008) On-chip counting the number and the percentage of CD4 + T lymphocytes. Lab Chip 8:309-315

WHO (2011) Chapter 7: microbial aspect. Guidelines for drinkingwater quality. Edn 4

WHO Guidelines for drinking-water quality (2006) Vol 1 recommendations. World Health Organization, Geneva

Yang S, Rothman RE (2004) PCR-based diagnostics for infectious diseases: uses, limitations, and future applications in acute-care settings. Lancet Infect Dis 4:337-348

Yaron S, Matthews K (2002) A reverse transcriptase-polymerase chain reaction assay for detection of viable Escherichia coli O157:H7: investigation of specific target genes. J Appl Microbiol 92:633-640

Zarlenga DS, Trout JM (2004) Concentrating, purifying and detecting waterborne parasites. Veter Parasitol 126:195-217

Zaytseva NV, Goral VN, Montagna RA, Baeumner AJ (2005) Development of a microfluidic biosensor module for pathogen detection. Lab Chip 5:805-811

Zhang L, Xua J, Mi L, Gong H, Jiang S, Yu Q (2012) Multifunctional magnetic-plasmonic nanoparticles for fast concentration and sensitive detection of bacteria using SERS. Biosens Bioelect 31:130-136

Zhao W, Yao S, Hsing IM (2006) Microsystem compatible strategy for viable Escherichia coli detection. Biosens Bioelectron 21:1163-1170

Zhu H, Mavandadi S, Coskun AF, Yaglidere O, Ozcan A (2011a) Optofluidic fluorescent imaging cytometry on a cell phone. Anal Chem 83:6641-6647

Zhu H, Yaglidere O, Su TW, Tseng D, Ozcana A (2011b) Costeffective and compact wide-field fluorescent imaging on a cellphone. Lab Chip 11:315-322 